JPE 12-4-15

\title{
Control Strategy Based on Equivalent Fundamental and Odd Harmonic Resonators for Single-Phase DVRs
}

\author{
Guofei Teng ${ }^{\dagger}$, Guochun Xiao*, Leilei Hu*, Yong Lu*, and Yuba Raj Kafle* \\ ${ }^{\dagger *}$ School of Electrical Engineering, State Key Laboratory of Electrical Insulation and Power Equipment, \\ Xi'an Jiaotong University, Xi'an, China
}

\begin{abstract}
In this paper, a digital control strategy based on equivalent fundamental and odd harmonic resonators is proposed for single-phase DVRs. By using a delay block, which can be equivalent to a bank of resonators, it rejects the fundamental and odd harmonic disturbances effectively. The structure of the single closed-loop control system consists of a delay block, a proportional gain and a set of zero phase notch filters. The principle of the controller design is discussed in detail to ensure the stability of the system. Both the supply voltage and the load current feedforwards are used to improve the response speed and the ability to eliminate disturbances. The proposed controller is simple in terms of its structure and implementation. It has good performances in harmonic compensation and dynamic response. Experimental results from a $2 \mathrm{~kW}$ DVR prototype confirm the validity of the design procedure and the effectiveness of the control strategy.
\end{abstract}

Key words: DVR, Equivalent resonators, Harmonic compensation, Single loop, Zero phase notch filter

\section{INTRODUCTION}

A dynamic voltage restorer (DVR) is one of the most effective methods to solve voltage sags and other voltage quality problems [1]. A control strategy is critical for the DVR system performance. In [2], the $\mathrm{H} \infty$ robust control strategy is used to enhance the system's ability to eliminate disturbances. However, better performance requires higher order controllers. Unfortunately, reducing the order of the system will also reduce the performance of the system. Proportional resonant (PR) control can obtain a high gain in the resonant frequency [3][4], but only selected harmonics are compensated. However, adding more resonators will increase the computational complexity of system. Moreover, a PR controller is very sensitive to discrete parameters [5], so it needs a specific method for implementation [4][6], which increases the implementation complexities. The traditional repetitive

Manuscript received Dec. 6, 2011; revised May 7, 2012

Recommended for publication by Associate Editor Kyo-Beum Lee.

${ }^{\dagger}$ Corresponding Author: tgf1253@stu.xjtu.edu.cn

Tel:+86-29-82666243, Fax:+86-29-82665223, Xi’an Jiaotong Univ.

*School of Electrical Engineering, State Key Laboratory of Electrical Insulation and Power Equipment, Xi'an Jiaotong University, China controller can improve the system steady-state performance and robustness, but its response is slow. As a result, it needs to be combined with instantaneous control methods [7][8]. Furthermore, the controller needs an additional second-order filter structure to ensure its stability [9].

In recent years, a delay block with feedback and feedforward has been used to compensate harmonics, which can be equivalent to a set of fundamental and odd harmonic resonators. It can also classified as repetitive controller [10]-[13]. This delay controller is used in active power filters (APF), static synchronous compensators (STATCOM) and other applications to obtain a strong harmonic suppression ability [10]-[13]. A controller used in the three-phase synchronous frame rejects the background harmonics while using a resonator in the stationary frame will increase the fundamental frequency resonator gain [13]. However, to ensure stability, a first order low-pass filter and a complex adaptive algorithm are involved.

\section{STRUCTURE OF SINGLE-PHASE DVR SYSTEMS}

The topology of a single-phase DVR is shown in Fig.1(a). In 


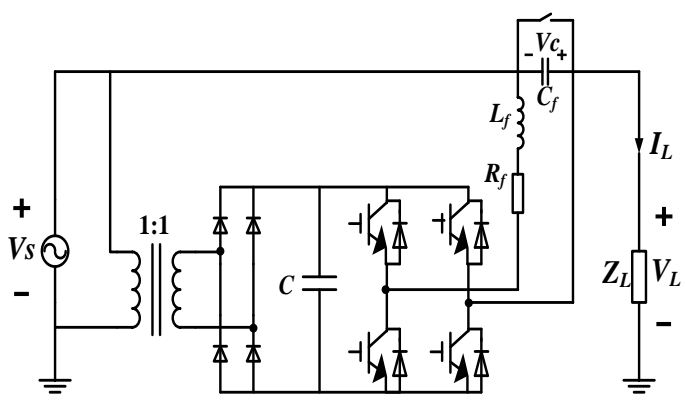

(a) Topology.

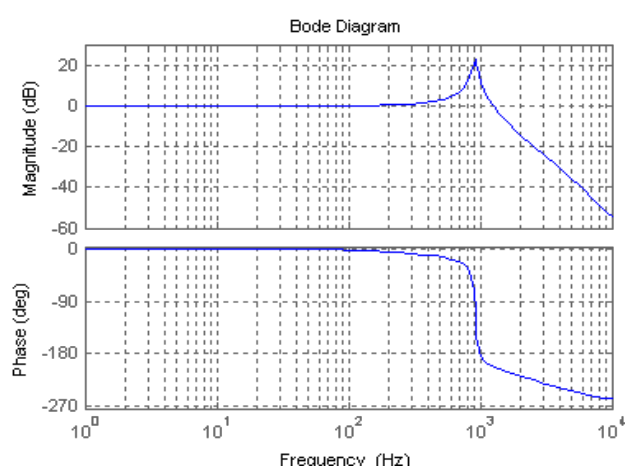

(b) Frequency response of $G_{\text {inr }}(\mathrm{s})$.

Fig. 1. A single-phase DVR system.

this figure, $V_{s}$ is the supply voltage, $V_{L}$ is the load voltage, $L_{f}$ and $C_{f}$ are the filter inductor and capacitor, respectively, and $R_{f}$ is the equivalent resistor of the filter inductor and inverter. The time delay resulting from the sampling and switching frequency of the inverter in a digital system is $T_{s}$. For simplicity, this can be replaced by a first-order element $1 /\left(T_{s} s+1\right)$ since the system switching frequency is much higher than that of the output. The inverter gain can be normalized to 1 in a digital control system. Therefore, considering the digital control delay and the equivalent resistance, the transfer function of the controlled object can be obtained by (1). A typical Bode plot is shown in Fig.1 (b).

$$
G_{i n v}(s)=\frac{1}{T_{s} s+1} \frac{1}{L_{f} C_{f} s^{2}+C_{f} R_{f} s+1}
$$

\section{CONTROL STRATEGY ANALYSIS}

\section{A. Equivalent Fundamental and Odd Harmonic Resonators Controller}

One kind of the delay controller involving negative feedback and negative feedforward is presented in Fig.2 [10]. The transfer function of the controller can be obtained by:

$$
\frac{Y(s)}{E(s)}=\frac{1-e^{-s T_{d}}}{1+e^{-s T_{d}}}
$$

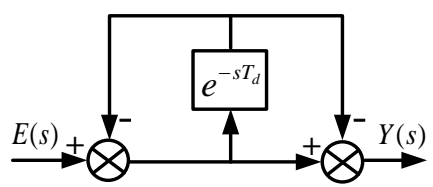

Fig. 2. Structure of delay block with negative feedback and negative feedforward.

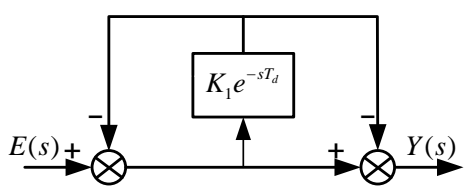

Fig. 3. Delay controller block after introduction of $K_{1}$.

Where $T_{d}=\frac{1}{n} T_{0} \quad(\mathrm{n}=1,2,3 \ldots)$ is the delay time, and $T_{0}=2 \pi / \omega_{0}$ is the fundamental period. From [14], (2) can be expressed as:

$$
\begin{aligned}
\frac{Y(s)}{E(s)} & =\frac{e^{s T_{d} / 2}-e^{-s T_{d} / 2}}{e^{s T_{d} / 2}+e^{-s T_{d} / 2}} \\
& =\frac{n}{2} \frac{4 \omega_{0}}{\pi} \sum_{k=1}^{\infty} \frac{s}{s^{2}+\left[\frac{n}{2}(2 k-1)\right]^{2} \omega_{0}^{2}}
\end{aligned}
$$

It can be seen in (3) that when $\mathrm{n}=2, T_{d}=T_{0} / 2$, then (4) can be obtained as:

$$
\frac{Y(s)}{E(s)}=\frac{4 \omega_{0}}{\pi} \sum_{k=1}^{\infty} \frac{s}{s^{2}+(2 k-1)^{2} \omega_{0}^{2}}
$$

It is shown in (4) that by setting the delay time to $1 / 2$ of a fundamental period, the delay controller can be regarded as a bank of resonators, which can compensate both the fundamental and odd harmonics. As the disturbances in a single-phase system contain mainly odd harmonics, the delay controller can be directly used to achieve compensation of the harmonics.

\section{B. Design of the Delay Block Attenuation Factor $K_{1}$}

The resonant gain of the resonators at frequency $(2 k-1) \omega_{0}$, shown in (4), is infinite which is difficult to achieve. Therefore, an attenuation factor $K_{1}\left(K_{1}<1\right)$ should be multiplied by the delay block shown in Fig.2 [10]. With $K_{1}$ included, both the bandwidth and the robustness are improved greatly. A controller block diagram of this is presented in Fig. 3.

$K_{1}$ can be written in the exponential form as $K_{1}=e^{-\sigma T_{d}}$, where $T_{d}=T_{0} / 2$. Thus from (4), the following equation can be obtained: 


$$
\begin{aligned}
G_{R e}(s) & =\frac{Y(s)}{E(s)}=\frac{1-K_{1} e^{-s T_{d}}}{1+K_{1} e^{-s T_{d}}}=\frac{1-e^{-(s+\sigma) T_{d}}}{1+e^{-(s+\sigma) T_{d}}} \\
= & \frac{4 \omega_{0}}{\pi} \sum_{k=1}^{\infty} \frac{(s+\sigma)}{s^{2}+2 \sigma s+\sigma^{2}+\left[(2 k-1) \omega_{0}\right]^{2}}
\end{aligned}
$$

It can be seen from (5) that as $K_{1}$ is added into the delay block, the denominator of controller transfer function has an additional first-order $2 \sigma \mathrm{s}$ element, which is equivalent to the addition of damping to an ideal resonator. Thus the design of $K_{1}$ is analogous to the design method of a quasi-resonance. From [15], a quasi-resonator has an expression as:

$$
g_{0}(s)=\frac{2 K_{r} \omega_{c u t} s}{s^{2}+2 \omega_{c u t} s+\omega_{h}{ }^{2}}
$$

where, $K_{r}$ is the gain of the quasi-resonators, $\omega_{\text {cut }}$ is the cutoff frequency and $\omega_{h}$ is the resonant frequency. By comparing equations (5) and (6), it can be seen that the purpose of the first-order term $2 \sigma s$ in (5) is the same as that of $2 \omega_{\text {cut }} s$ in (6), which is to increase the damping of the controller. The role of $\sigma$ is similar to that of $\omega_{\text {cut }}$. For (5), $\sigma$ in the numerator just affects the amplitude of the equivalent resonators at each resonant frequency. Because of the tiny effect of the numerator of (5), $\sigma$ can be ignored. The influence of $\sigma^{2}$ on the resonant frequencies $\left[(2 k-1) \omega_{0}\right]^{2}$ is less than $10^{-4} \mathrm{rad} / \mathrm{s}$ in the denominator, which can also be neglected. Therefore, the introduction of $\sigma$ in (5) only influences the damping of the controller while its influence on the resonant frequencies can be ignored, thus (5) can be approximated by:

$$
G_{R e}(s) \approx \frac{4 \omega_{0}}{\pi} \sum_{k=1}^{\infty} \frac{s}{s^{2}+2 \sigma s+\left[(2 k-1) \omega_{0}\right]^{2}}=G_{r}(s)
$$

Therefore, $\sigma$ in (7) is similar to $\omega_{\text {cut }}$ in (6). It also affects the resonator gain and bandwidth. Let $s=j \omega$, substitute in (7), and it can be obtained by:

$$
\begin{aligned}
G_{r}(j \omega) & =\frac{4 \omega_{0}}{\pi} \sum_{k=1}^{\infty} \frac{j \omega}{-\omega^{2}+2 \sigma \omega j+[(2 k-1)]^{2} \omega_{0}^{2}} \\
& =\sum_{k=1}^{\infty} \frac{K_{h}}{1+\left[(2 k-1)^{2} \omega_{0}^{2}-\omega^{2}\right] /(2 \sigma \omega j)}
\end{aligned}
$$

where $K_{h}=\frac{2 \omega_{0}}{\pi \sigma}$, the bandwidth of the equivalent resonator is then defined as:

$$
\left|G_{r}(j \omega)\right|=\frac{K_{h}}{\sqrt{2}}
$$

Let $\left|\left[(2 k-1)^{2} \omega_{0}^{2}-\omega^{2}\right] /(2 \sigma \omega j)\right|=1$, then the relationship between $\sigma$ and the bandwidth (BW) can be obtained by $B W=\sigma /(2 \pi) \mathrm{Hz}$.

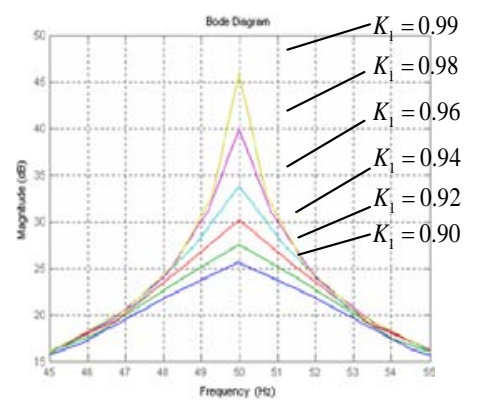

(a) Influence of $K_{1}$ on the resonator gain.

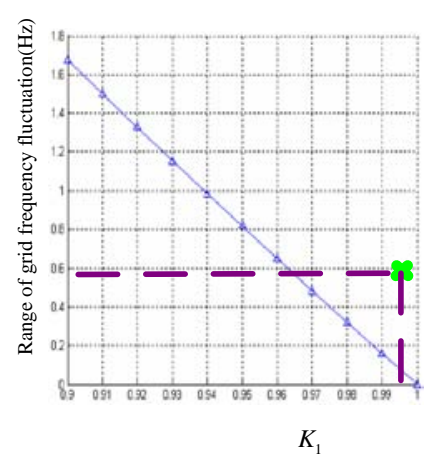

(b) Relationship between $K_{1}$ and bandwidth .

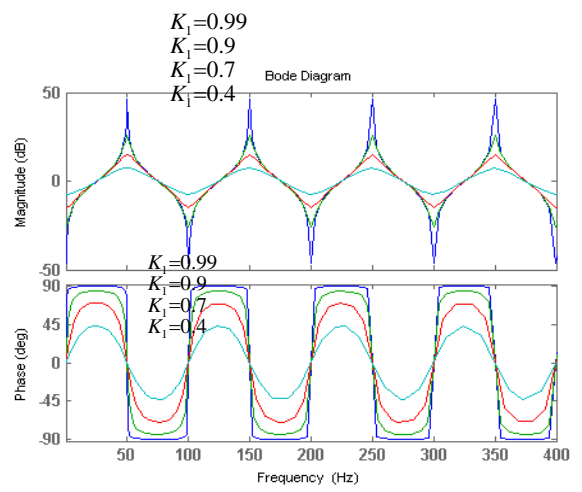

(c) Influence of $K_{1}$ on the resonator frequency response. Fig. 4. Influence of $K_{1}$ on the resonator performance.

Generally, the frequency deviation in the limit of $\pm 0.2 \mathrm{~Hz}$ is allowable in a normal power system. When the system power is small, the limit can be extended to $\pm 0.5 \mathrm{~Hz}$. To obtain good robustness, the bandwidth of the resonant controller should at least be greater than $0.5 \mathrm{~Hz}$, that is $\sigma>\pi$. According to the relationship between $K_{1}$ and $\sigma, K_{1}$ should be less than 0.97 . On the other hand, $K_{1}$ affects the controller gain at the resonant peak. For different values of $K_{1}$, the corresponding resonator gain, the bandwidth, and the frequency characteristics of the resonators $G_{R e}(s)$ are shown in Fig.4(a),(b),(c), respectively. 


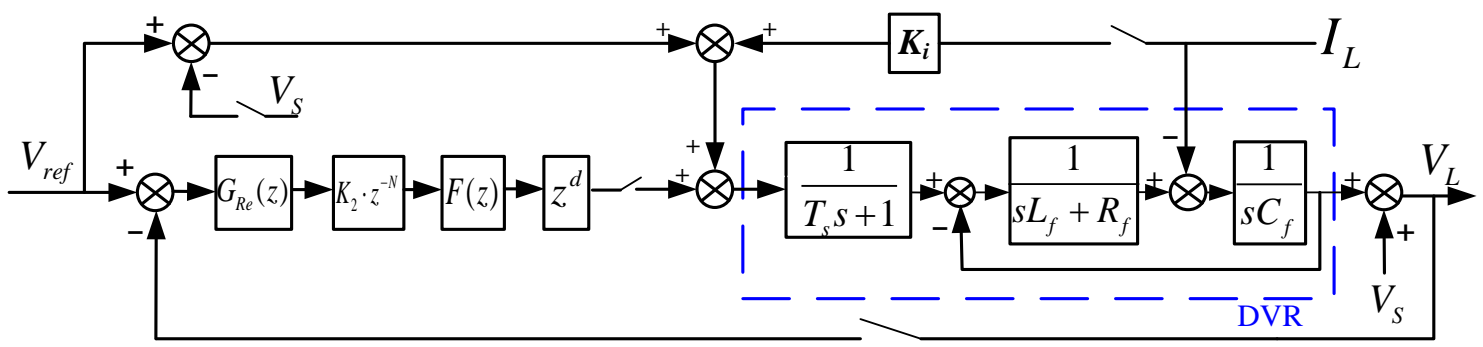

Fig. 5. Proposed controller based on the equivalent fundamental and odd harmonic resonators for a single-phase DVR.

From Fig.4 (a), to get the resonator gain as high as possible and to fulfill the bandwidth requirement, $K_{1}$ is designed as 0.96 , which means that $\sigma=4$. Then the equivalent resonator gain in equation (8) is $K_{h}=\frac{2 \omega_{0}}{\pi \sigma}=\frac{2 \times 100 \pi}{\pi \times 4}=50$, which is $34 \mathrm{~dB}$. The expression of the delay controller shown in Fig.3 is $G_{R e}(s)=\frac{1-0.96 e^{-s T_{0} / 2}}{1+0.96 e^{-s T_{0} / 2}}$, where the z-domain form of $e^{-s T_{0} / 2}$ is $z^{-N / 2}$. In this paper, the sampling switching frequency is $15 \mathrm{kHz}$ (see Table I) and $N$ equals 300 . Therefore, the form of the controller used in this paper can be expressed as:

$$
G_{R e}(z)=\frac{Y(z)}{E(z)}=\frac{1-0.96 z^{-150}}{1+0.96 z^{-150}}
$$

It can be written in the differential form as:

$$
y(k)=e(k)-0.96 e(k-150)-0.96 y(k-150)
$$

\section{CLOSED-LOOP CONTROL SYSTEM AND PARAMETERS DESIGN}

As mentioned above, the delay controller in (2) is equivalent to a series of resonant controllers at both the fundamental frequency and the odd harmonics. This controller can obtain a proper band width and resonant gain as the attenuation factor $K_{1}$ is well designed. Additionally, it is easy to realize in digital systems as mentioned in (11). Therefore, considering that a single-phase system mainly contains odd harmonics, a control strategy based on the delay controller for a single-phase DVR is proposed in this paper, as shown in Fig. 5. Where, $G_{\mathrm{Re}}(z)$ is the equivalent fundamental and the odd harmonic resonator. The proportional gain $K_{2}$ and the zero phase notch filters $F(z)$ are designed to ensure system stability. Since the expression of $F(z)$ contains the time advance unit, the implementation needs to introduce a one cycle delay $z^{-N}$. In addition, the time advance element $z^{d}$ for the phase delay compensation of $G_{i n v}(s)$ is based on $z^{-N}$. Meanwhile, the supply voltage and the load current feedforwards are added to reject the disturbances and to increase the response speed.

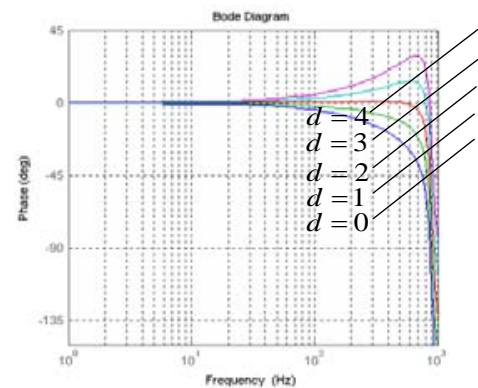

Fig. 6. Bode plot of $G_{i n v}(z) z^{d}$ with different $d$.

For the stability of the system, where the resonant frequency $\omega_{f}$ of the LC filters is seen as the cutoff point, the frequency response characteristic is divided into two parts for discussion. When $\omega>\omega_{f}$, setting the zero phase notch filter appropriately makes the gain of the whole system under $0 \mathrm{~dB}$ to ensure system stability. When $\omega<\omega_{f}$, by using another zero phase notch filter to eliminate the resonant peak of the LC filters, shown in Fig.1(b), so that the magnitude-frequency characteristic of the controlled object in the low frequency band is approximately in a line with $0 \mathrm{~dB}$. In addition, a time advance unit $z^{d}$ is used to compensate for the controlled object phase delay. The phase characteristics of $G_{i n v}(z) z^{d}$ with different values of $d$ are shown in Fig. 6.

From Fig.6, it can be seen that when $d=2$, the phase-frequency characteristic of the controlled object is approximate $0^{\circ}$ in the low frequency band. Although the compensated phase still has a small delay near the vicinity of $\omega_{f}$, the zero phase notch filter magnitude-frequency characteristic will have a higher attenuation near $\omega_{f}$ (a detailed discussion of this can be found in section 4.2), thus the impact of a small phase delay to the system can be ignored. Therefore, the frequency response characteristic of the controlled object $G_{i n v}(z) z^{d}$ can be approximated as the magnitude of $0 \mathrm{~dB}$ and the phase of $0^{\circ}$ in the low frequency band. Thus the impact of its frequency response characteristic 
on the system stability is negligible.

\section{A. Design of the Proportional Gain $K_{2}$}

The frequency response curves of the delay controller shown in (10) are similar to the one in Fig. 4 (c). The phase always changes periodically with a frequency of $-90^{\circ} \sim 90^{\circ}$. As the influence of the controlled object on the system can be essentially ignored at lower frequencies, the phase-frequency curve of the open-loop transfer function of the whole system before the LC filter resonant frequency will not cross $-180^{\circ}$, which means that the system is always stable.

However, to eliminate the resonance peak and to compensate the phase delay of the controlled object, this paper uses zero phase notch filters $F(z)$ (for details refer to the next section) and a time advance unit $z^{d}$. Though the zero phase notch filters will not bring an excess phase variation to the system, their expression also has a unit of time advance. Thus the realization needs to introduce a cycle delay $z^{-N}$.

The delay element $e^{-s T_{0}}$ will affect the phase of the resonator banks, and its frequency domain will be in the form:

$$
e^{-j \omega T_{0}}=1 \angle\left(-\omega T_{0}\right)=\operatorname{co}\left(s-\omega T_{0}\right)+j \sin \left(-\omega T_{0}\right)
$$

The relationship between its phase $\alpha$ and frequency can be derived as follows:

$$
\alpha=-\omega T_{0}=-\frac{2 \pi \omega}{\omega_{0}}
$$

Defining that:

$$
G_{C 0}(z)=G_{\mathrm{Re}}(z) z^{-N}
$$

The phase-frequency curve will pass through $-\pi,-3 \pi$, $\begin{array}{llll}-5 & \pi & \ldots\end{array}$ Its frequency domain is expressed as $G_{C 0}(j \omega)=G_{\mathrm{Re}}(j \omega) e^{-j \omega T_{0}}$. Take the equivalent fundamental component resonator for example. It can be calculated that when $\left|G_{C 0}(j \omega)\right|=0 \mathrm{~dB}$, the corresponding phase angle of $G_{C 0}(j \omega)$ is less than $-180^{\circ}$. As a result, the phase margin is less than 0 and the system will be unstable. This indicates that a one cycle delay $e^{-s T_{0}}$ brings another stability problem.

Therefore, this paper presents a proportional element in series to ensure the stability of the controller. The proportional gain is assumed as $K_{2}$. The form of the controller after introduction of the proportional element is as follows:

$$
G_{C}(z)=K_{2} G_{\mathrm{Re}}(z) z^{-N}
$$

As mentioned above, the controlled object, after using the notch filter and phase compensation, does not affect the lowfrequency characteristics of the system. Thus the stability of the system only depends on the stability of the controller $G_{C}$.

From Fig. 4(c) and the analysis above, it can be seen that $G_{\mathrm{Re}}$ has a phase jump at each resonant frequency. It can also be seen that the phase-frequency characteristic of $G_{\mathrm{Re}}$ is close to $\pi / 2$ before the phase jump, and after that it is close to $-\pi / 2$.
Therefore, let the phase angle of $G_{\mathrm{Re}}$ before and after the phase jump be $\pi / 2-\beta$ and $-\pi / 2+\beta$ respectively, where $\beta$ is a small positive angle. From (13) it can be seen that when $\omega=\omega_{0}$, the phase delay is $\alpha=-2 \pi$. For the equivalent fundamental resonator, the stability condition of $G_{C}(j \omega)$ is that when the phase frequency curve crosses $-\pi$ and $-3 \pi$, the gain of $G_{C}(j \omega)$ should be less than zero, that is: $\left|G_{C}(j \omega)\right|<0 d B$. This means that the angular frequency should satisfy the following equations:

$$
\begin{aligned}
& -2 \pi \omega / \omega_{0}+\pi / 2-\beta=-\pi \\
& -2 \pi \omega / \omega_{0}-\pi / 2+\beta=-3 \pi
\end{aligned}
$$

If $\pi / 2-\beta \approx \pi / 2$ and $-\pi / 2+\beta \approx-\pi / 2$, then the designed proportional gain $K_{2}$ after this approximation will surely meet the condition $\left|G_{C}(j \omega)\right|<0 d B$ with a certain margin. Then the angular frequencies relationships are approximated by:

$$
\begin{aligned}
& -2 \pi \omega / \omega_{0}+\pi / 2=-\pi \\
& -2 \pi \omega / \omega_{0}-\pi / 2=-3 \pi
\end{aligned}
$$

By solving the equations above, results can be obtained as $\omega=3 \omega_{0} / 4$ and $\omega=5 \omega_{0} / 4$, when the phase frequency curve of $G_{C}(j \omega)$ crosses the points of $-\pi$ and $-3 \pi$, respectively. Combined with the condition $\left|G_{C}(j \omega)\right|<0 d B$, the range of the proportional gain $K_{2}$ can be obtained as $0<K_{2}<0.415$.

Extended to the $(2 k-1) \omega_{0}$ harmonic resonators, the same range of $K_{2}$ can be obtained. In fact, the magnitude-frequency characteristic curve of the controller $G_{C}(j \omega)$ is symmetrical to each resonant frequency and it is independent of the number of the resonant frequency $2 k-1$.

In addition, through a further analysis, the relationship between the proportional gain $K_{2}$ and the attenuation coefficient $K_{1}$ can be obtained when the value of $\sigma$ is small. Equation (8) can be re-written as:

$$
\begin{aligned}
& G_{r}(j \omega)= \\
& \sum_{k=1}^{\infty} \frac{K_{h}}{\sqrt{1^{2}+\left\{\left[(2 k-1)^{2} \omega_{0}^{2}-\omega^{2}\right] /(2 \sigma \omega)\right\}^{2}}} \angle \tan ^{-1}\left(\frac{(2 k-1)^{2} \omega_{0}^{2}-\omega^{2}}{2 \sigma \omega}\right)
\end{aligned}
$$

The magnitude-frequency characteristic curve of $G_{C}(j \omega)$ is symmetrical to each resonant frequency, and it is independent of the number of resonators. Therefore, the fundamental component resonator is taken as an example, which means that $k$ equals 1 . Also, from equation (7), the approximation $G_{R e}(s) \approx G_{r}(s)$ can be obtained, thus the following equation can be obtained by: 


$$
\begin{aligned}
G_{C} & (j \omega) \approx K_{2} G_{r}(j \omega) e^{-j \omega T_{0}} \\
& =\frac{K_{h} K_{2}}{\sqrt{1^{2}+\left[\left(\omega_{0}^{2}-\omega^{2}\right) / 2 \sigma \omega\right]^{2}}} \angle\left[\tan ^{-1}\left(\frac{\omega_{0}^{2}-\omega^{2}}{2 \sigma \omega}\right)-\omega T_{0}\right]
\end{aligned}
$$

For (21), the magnitude-frequency characteristic is $\left|G_{C}(j \omega)\right|=\frac{K_{h} \cdot K_{2}}{\sqrt{1^{2}+\left[\left(\omega_{0}^{2}-\omega^{2}\right) / 2 \sigma \omega\right]^{2}}} \quad, \quad$ and the phase-frequency characteristic $\varphi=\angle\left[\tan ^{-1}\left(\frac{\omega_{0}^{2}-\omega^{2}}{2 \sigma \omega}\right)-\omega T_{0}\right]$. According to the stability conditions of the system, that is, when $\varphi=-\pi$, $\left|G_{C}(j \omega)\right|<0 d B$. Then, the following equation can be obtained when the system is stable:

$$
K_{2}<\frac{\pi}{\omega_{0}} \sqrt{\sigma^{2}+\left[\frac{\left(\omega_{0}^{2}-\omega^{2}\right)}{2 \omega}\right]^{2}}=\frac{1}{200} \sqrt{\sigma^{2}+8387}
$$

From (22), it can be seen that when the value of $K_{1}$ is near to $1, \sigma$ is very small so that its impact on the $K_{2}$ is negligible. That means, the design of $K_{2}$ will not be influenced much by $K_{1}$. In addition, as $K_{1}$ directly effects the resonant gain of the equivalent resonators, it can be seen that the design of $K_{2}$ is irrelevant to the resonator gain when $\sigma$ is small.

The gain margin and phase margin of $G_{C}(j \omega)$ under different values of $K_{2}$ are shown in Fig.7. In general, the phase margin of the control system should be larger than $30^{\circ}$, and the gain margin should be larger than $6 \mathrm{~dB}$. In this paper, $K_{2}$ is chosen to be 0.22 . Then the gain of the equivalent resonator banks is $K_{2} K_{h}=11$, that is $20.8 \mathrm{~dB}$. In this case, the frequency response of $G_{C}(j \omega)$ is shown in Fig. 8.

\section{B. Design of the Zero Phase Notch Filter}

The peak resonance of $G_{i n v}(\mathrm{~s})$, shown in Fig. 1(b), will affect the stability of the system. Generally, voltage or current state feedback is adopted to increase the damping to get a stable system [8]. For the use of equivalent resonators banks, [10]-[12] is based on an adaptive algorithm which can adjust the parameters automatically within a certain range to ensure system stability. In this paper, to simplify the structure of the control, zero phase shift notch filters, $F_{1}$ and $F_{2}$, are used.

A zero phase notch filter will bring almost no additional phase shift to the system[16], and its structure is shown as:

$$
F_{0}(z)=\frac{z^{m}+a_{0}+z^{-m}}{2+a_{0}}
$$

where $a_{0}$ and $\mathrm{m}$ are constants, whose values are related to the location and shape of the notch filter.

The relationship between the discrete domain and the frequency domain is $Z=e^{j \omega T_{s}}=e^{j \theta}$. Combined with (23), the equation can be obtained as follow:

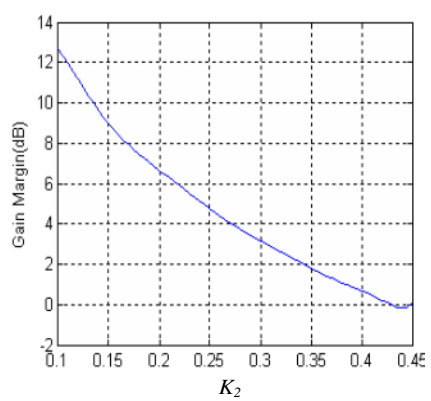

(a) Gain margin.

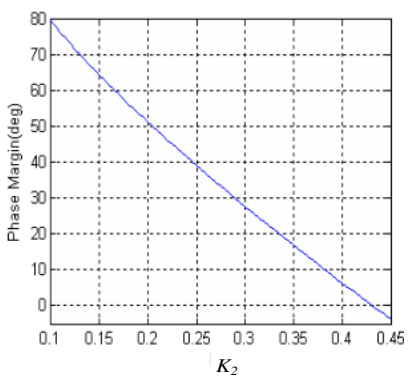

(b) Phase margin.

Fig. 7. Stability margin of $G_{C}(j \omega)$ with different $K_{2}$.

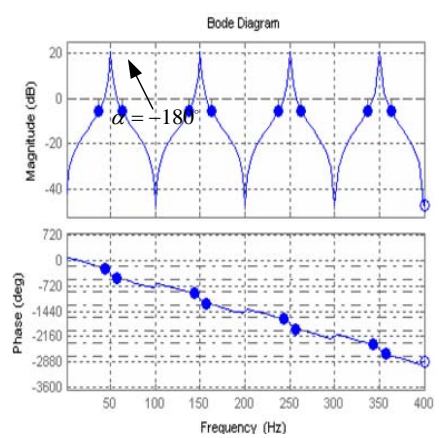

Fig. 8. Frequency response of $G_{C}(j \omega)$.

$$
F_{0}(\theta)=\frac{e^{j m \theta}+a_{0}+e^{-j m \theta}}{2+a_{0}}=\frac{2 \cos m \theta+a_{0}}{2+a_{0}}
$$

When $a_{0}=2$ and $F_{0}(\theta)=0, F_{0}(z)$ has the largest attenuation. Under this condition, $\theta$ should fulfill the equation: $2 \cos m \theta+2=0$, which is the same as $m \theta=(2 k-1) \pi,(\mathrm{k}=1,2 \ldots)$. It is easy to see that the zero phase notch filter has more than one notch frequency, and that the first one $(k=1)$ is mainly considered for the $F(z)$ design.

First, the notch filter $F_{1}$ is used to eliminate the peak resonance, as shown in Figure 1(b). Therefore, the first notch frequency of $F_{1}$ is set to equal the resonant frequency of the LC filters, that is $\omega_{1}=\omega_{f}=1 / \sqrt{L_{f} C_{f}}$. Combined with (24) and the relation $\theta=\omega T_{s}$, it can be seen that: 


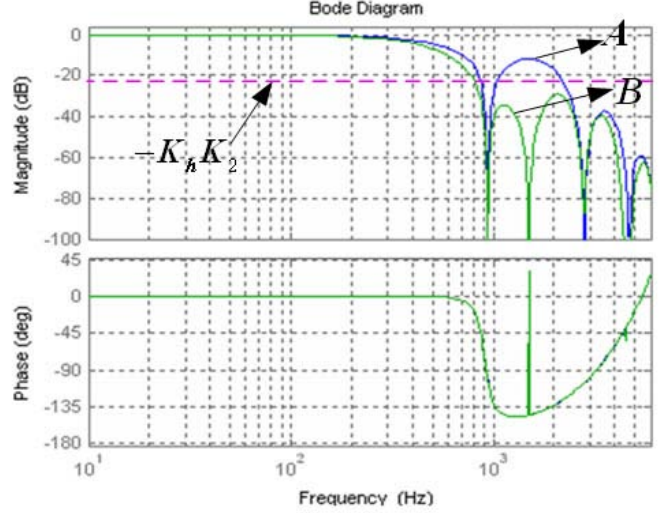

Fig. 9. Bode diagram of controlled object by adding notch filters.

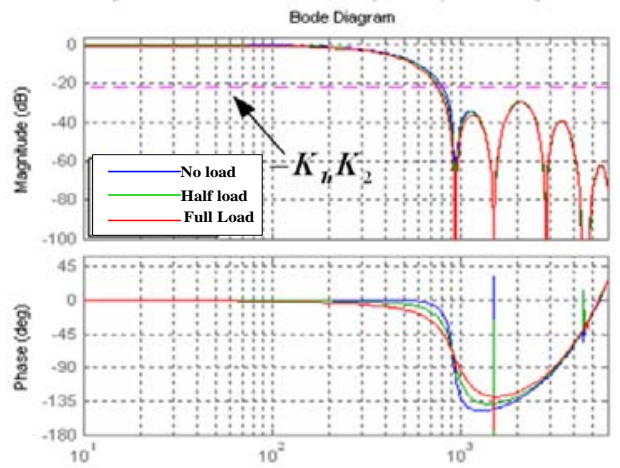

Fig. 10. Frequency responses with different loads of the controlled object by adding notch filters.

$$
m_{1}=\frac{\pi}{\omega_{f} T_{s}}=\frac{\pi}{T_{s}} \cdot \sqrt{L_{f} C_{f}}
$$

From the parameters shown in Table I, $m_{1}$ can be obtained as: $m_{1}=8.16$, rounded to $m_{1}=8$. Therefore, the expression of $F_{1}$ can be given as follows:

$$
F_{1}(z)=\frac{z^{8}+2+z^{-8}}{4}
$$

The notch filter has more than one notch frequency. These frequencies are at $(2 k-1) \omega_{f},(\mathrm{k}=1,2, \ldots)$. A Bode plot of the controlled object $G_{i n v}(z) z^{d}$ with $F_{1}$ added is presented as curve A in Fig. 9. It can be seen that the peak resonance of the LC filter is completely suppressed. In addition to $G_{i n v}(z) z^{d} F_{1}$, the open-loop frequency characteristic of the system contains the controller $G_{C}(j \omega)$. Since the magnitude-frequency characteristic of $G_{C}(j \omega)$ is like a bank of infinite resonators with the gain $K_{h} K_{2}=20.8 d B$, when the frequency is not much higher than $\omega_{f}$, the attenuation of the LC filters is insufficient. As a result, in the open-loop system, there are still some frequencies whose gains are larger than $0 \mathrm{~dB}$ when $\omega>\omega_{f}$. At the same time, when compared with the condition where $\omega<\omega_{f}$, it can be seen from curve A, in Fig.9,

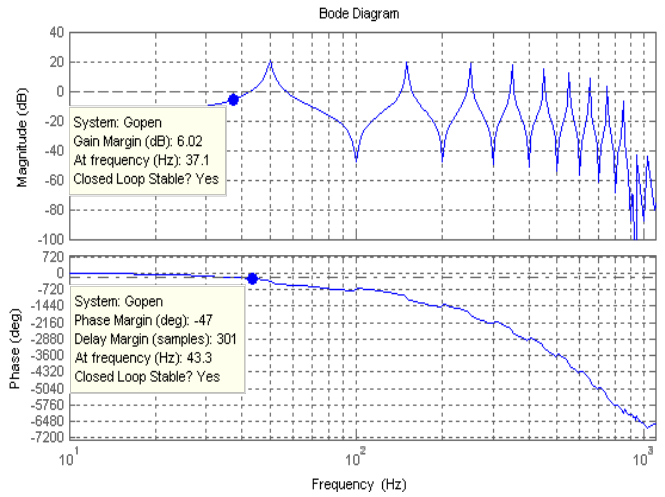

(a) Bode plot.

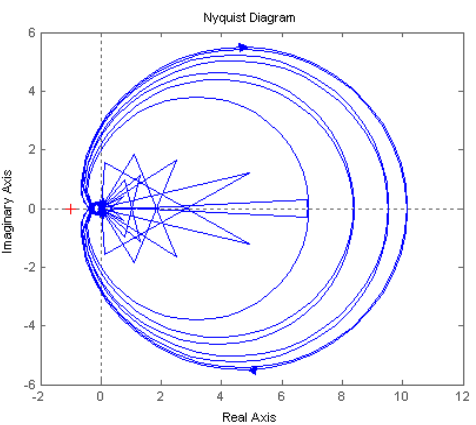

(b) Nyquist plot.

Fig. 11. Open-loop frequency response of the system with no-load connected.

that there is a much greater phase delay when $\omega>\omega_{f}$, which brings new instability factors. However, if the magnitude-frequency characteristic can be declined under 0dB, the problem will be solved. For this purposes, another zero phase notch filter $F_{2}$ is necessary.

It is easy to see that the gain of $F_{1}$, between $\omega_{f}$ and $3 \omega_{f}$, is close to $0 \mathrm{~dB}$, which will result in insufficient attenuation at the resonant frequencies of the equivalent resonators. Therefore, the notch frequency of $F_{2}$ should be selected near $\omega_{2}=\left(\omega_{f}+3 \omega_{f}\right) / 2=2 \omega_{f}$. According to (25), $m_{2}$ can be obtained as 4.08. Since the attenuation of the LC filter increases along with the frequency, the notch frequency of $F_{2}$ should be set at a lower value, which means that a larger value should be chosen for $m_{2}$. Therefore, $m_{2}$ is designed as 5 , and the expression of $F_{2}$ can be derived as:

$$
F_{2}(z)=\frac{z^{5}+2+z^{-5}}{4}
$$

After adding the group of notch filters $F(z)=F_{1}(z) F_{2}(z)$, the frequency response of $F(z) G_{i n v}(z) z^{d}$ is shown as the curve B, in Fig.9. It can be seen that when $\omega>\omega_{f}$, the magnitude-frequency characteristic curve of $F(z) G_{i n v}(z) z^{d}$ is always below $-K_{h} K_{2}$ with a small gain margin. This means 
TABLE I

MAIN PARAMETERS

\begin{tabular}{|c|c|}
\hline Rated output Voltage & $220[\mathrm{~V}]$ \\
\hline Supply voltage frequency & $220[\mathrm{~V}]$ \\
\hline Filter inductor & $1.5[\mathrm{mH}]$ \\
\hline Filter capacitor & $20[\mu \mathrm{F}]$ \\
\hline Equivalent filter resistance & $0.6[\Omega]$ \\
\hline Linear load & $22[\Omega]$ \\
\hline Dc link capacitor & $4700[\mu \mathrm{F}]$ \\
\hline Sampling frequency & $15000[\mathrm{~Hz}]$ \\
\hline
\end{tabular}

that when $\omega>\omega_{f}$, although the phase-frequency characteristic will cross through $(-2 k-1) \cdot 180^{\circ},(k=1,2, \ldots)$, the open-loop gain is invariably less than $0 \mathrm{~dB}$, which ensures the stability of the system. Combined with the analysis when $\omega<\omega_{f}$, the stability of the whole system is guaranteed.

As $F(z)$ is added, the frequency responses of the controlled object with different load conditions (including no load, half load and full load conditions), are described in Fig.10. Under the full load condition, the resistance is $22 \Omega$. Fig.10 proves that under different load conditions, the system has sufficient attenuation at high frequencies without unwanted resonance peaks.

An open-loop Bode plot of the system under no-load is shown in Fig.11(a). Using MATLAB, the smallest stability margin can be obtained near the fundamental frequency. The plot shows that the minimum gain margin is $6.02 \mathrm{~dB}$ and that the minimum phase margin is $47^{\circ}$. An open-loop Nyquist plot of the system under no-load is shown in Fig.11(b). Fig.11(b) indicates that the curve does not surround the point $(-1,0 \mathrm{j})$. At the same time, $G_{c}(z), F(z)$, and $G_{i n v}(z)$ have no open-loop right poles, which proves that the DVR control system is stable.

\section{EXPERIMENTAL VERIFICATION}

In a single-phase 2kVA DVR prototype, the effectiveness and practicality of the proposed control strategy are verified. The topology is shown in Fig. 1. The processor for the digital system is a DSP TMS320F2812, and the main experimental parameters are listed in Table I.

Fig. 12 presents the waveforms under the conditions of voltage sags and fluctuations with a resistive load, where $\mathrm{CH} 1$ is the supply voltage $V_{s}$, $\mathrm{CH} 2$ is the load voltage $V_{L}$ and $\mathrm{CH} 3$ is the load current $I_{L}$. Fig. 12(a) shows the compensation results for the voltage sag compensation. When the supply

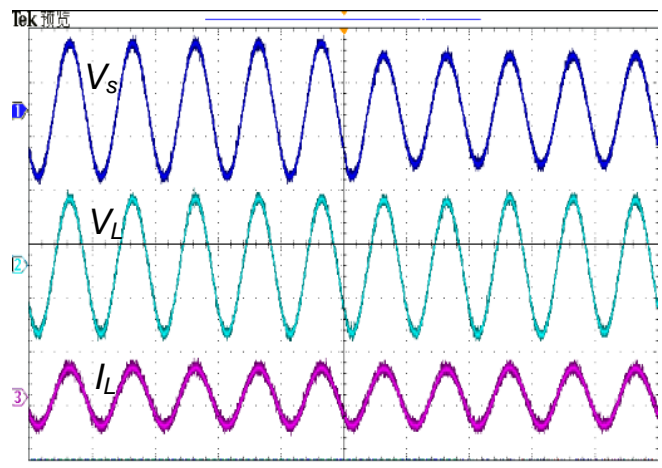

(a) Voltage sag (20ms/div).

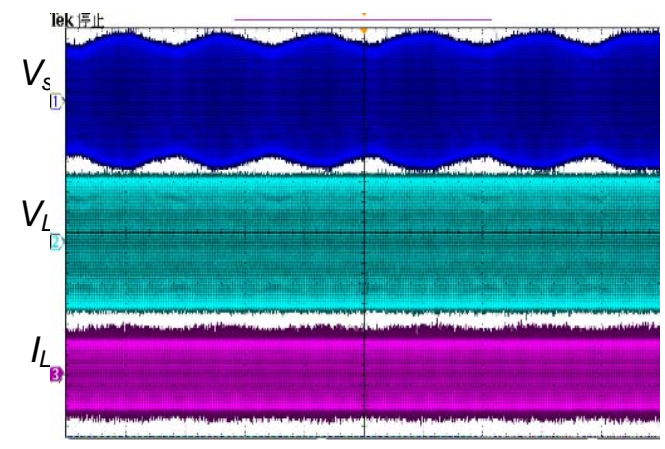

(b) Voltage fluctuation(2s/div).

Fig. 12. Compensation waveforms under voltage sag and fluctuation (CH1: supply voltage $V_{s}, 250 \mathrm{~V} / \mathrm{div}$; $\mathrm{CH} 2$ : load voltage $V_{L}, 250 \mathrm{~V} / \mathrm{div}$;CH3: load current $I_{L}, 25 \mathrm{~A} / \mathrm{div}$.

voltage dips from $220 \mathrm{~V}$ down to $180 \mathrm{~V}$, the load voltage is maintained in the range of $219 \sim 221 \mathrm{~V}$, the steady-state error is less than 1\%, and the THD is less than 1\%. Fig. 12(b) describes that when the supply voltage fluctuates from $180 \mathrm{~V}$ to $220 \mathrm{~V}$, the load voltage is almost maintained at $220 \mathrm{~V}$ with a THD of less than $1 \%$.

Fig. 13 presents the performances of the harmonic compensation under a linear load. In Fig. 13(a), the supply voltage is $200 \mathrm{~V}$ with $5^{\text {th }}$ and $7^{\text {th }}$ order harmonics, and a $\mathrm{THD}=20.8 \%$. After compensation the value of load voltage is $221 \mathrm{~V}$, and the THD declines to $1.2 \%$. In the condition of Fig. $13(\mathrm{~b})$, the supply voltage is $200 \mathrm{~V}$ with $3^{\text {rd }}, 5^{\text {th }}$, and $9^{\text {th }}$ order harmonics, and a $\mathrm{THD}=10.5 \%$. After compensation, the load voltage is $220.8 \mathrm{~V}$ with a $\mathrm{THD}=1.1 \%$. These results confirm that the proposed controller has a good odd harmonic compensation performance.

In Fig.14, the transient response to a load change under a $180 \mathrm{~V}$ sag condition is presented. During the process of load change, the load voltage remains stable at the value of $220 \mathrm{~V}$, indicating that the control method has a strong ability to reject load disturbances. 


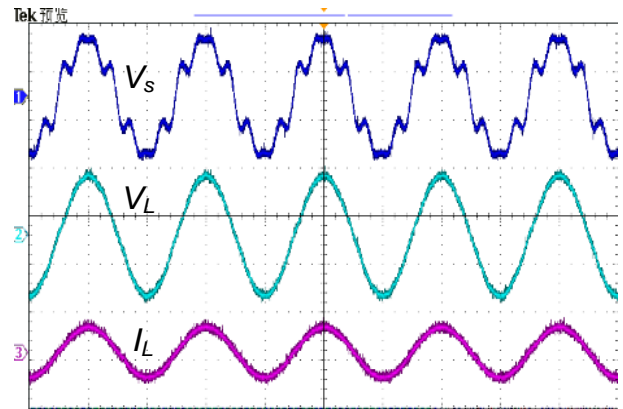

(a) Supply voltage with 5, 7 harmonics.

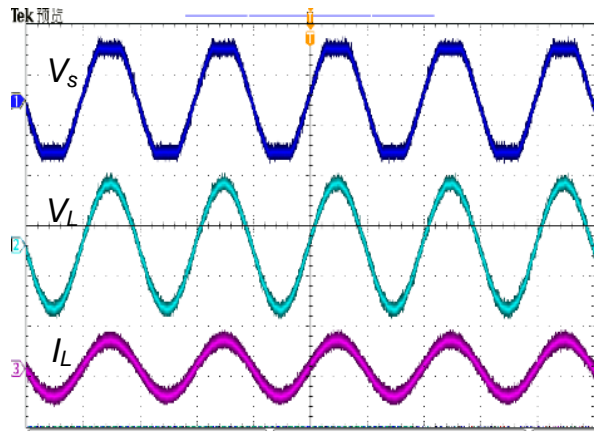

(b) Supply voltage with 3, 5, 9 harmonics.

Fig. 13 Odd harmonics compensation under different supply.

(CH1: supply voltage $V_{s}, 250 \mathrm{~V} / \mathrm{div}$; $\mathrm{CH} 2$ : load voltage $V_{L}$, 250V/div; CH3: load current $I_{L}, 25 \mathrm{~A} / \mathrm{div} ; \mathrm{t}: 10 \mathrm{~ms} / \mathrm{div}$ )

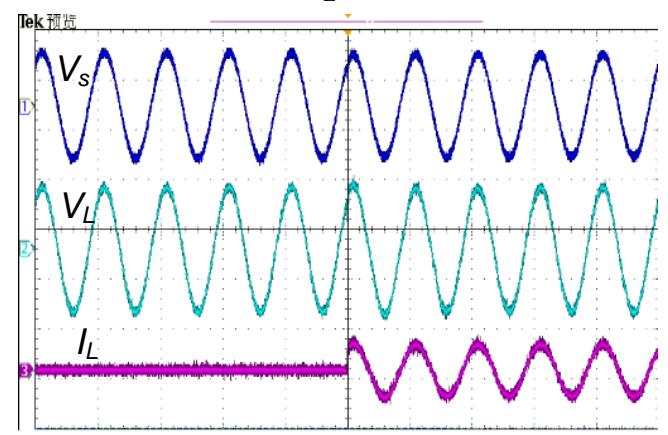

Fig. 14. Transient response to linear load added (CH1: supply voltage $V_{s}, 250 \mathrm{~V} /$ div; CH2: load voltage $V_{L}, 250 \mathrm{~V} / \mathrm{div}$; CH3: load current $I_{L}, 25 \mathrm{~A} / \mathrm{div}$; t::20ms/div).

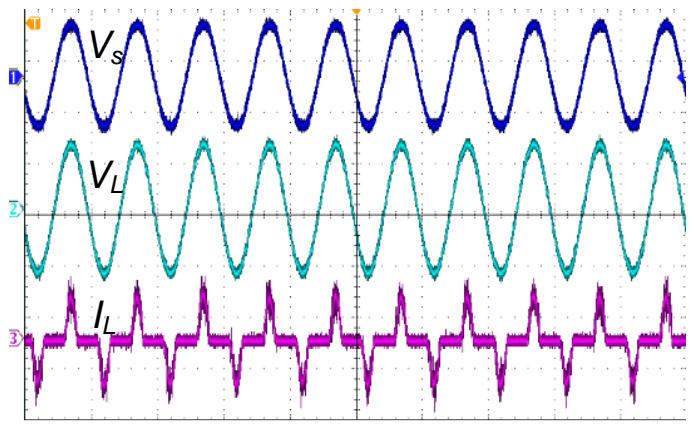

Fig. 15. Compensation for supply voltage sag with a nonlinear load(CH1: supply voltage $V_{s}, 250 \mathrm{~V} /$ div; CH2: load voltage $V_{L}$, 250V/div; CH3; load current $I_{L}$, 20A/div; t:20ms/div).
Fig.15 shows the compensation waveforms when supply voltage sags occurs at $180 \mathrm{~V}$ under a non-linear load. The results demonstrate that the system can effectively eliminate non-linear load disturbances. The value of the load voltage is between $219 \sim 221 \mathrm{~V}$, and the THD is $1 \%$.

\section{CONCLUSIONS}

In this paper, a new control strategy based on equivalent fundamental and odd harmonic resonator banks is proposed for single-phase DVRs. The analysis and design of the parameters in the controller are discussed in detail in this paper. By properly setting the attenuation coefficient and the proportional gain along with a group of zero phase notch filters, closed loop system stability and steady-state accuracy can be obtained. The transient response and the ability to reject disturbances can be increased by using supply voltage and load current feedforwards. This control scheme is just a single loop with double feedforwards. Thus it is very easy to implement. The proposed control scheme can solve the problems of the limitations of multiple harmonics compensation and the digital implementation of a general resonant controller. Moreover, the limitations caused by the complexity of the adaptive algorithm will be avoided. Experimental verification of the controller is carried out on a $2 \mathrm{~kW}$ DVR prototype, and the experimental results show that the proposed controller has a high compensation precision, strong harmonic suppression, a faster dynamic response, and simple realization, thus it has good engineering practicality.

\section{ACKNOWLEDGMENT}

This paper and its related research work are supported by national Natural Science Foundation of China (NSFC) (project no. 50877065)

\section{REFERENCES}

[1] N. G. Hingorani. "Introducing Custom Power," IEEE Spmtrum, pp.41-48, Jun. 1995.

[2] Y. W. Li, D. M. Vilathgamuwa and F. Blaabjerg, “A robust control scheme for medium- voltage-level DVR implementation,” IEEE Trans. Ind. Electron.. Vol. 54, No. 4, pp. 2249-2261, Aug. 2007.

[3] A. G. Yepes, F. D. Freijedo, and O. Lopez, "High-performance digital resonant controllers implemented with two integrators," IEEE Trans. Power Electron., Vol. 26, No. 2, pp. 563-576, Feb. 2011.

[4] I. J. Gabe, V. F. Montagner, and H. Pinheiro, "Design and implementation of a robust current controller for VSI connected to the grid through an LCL filter," IEEE Trans. Power Electron., Vol. 24, No. 6, pp. 1444-1452, Jun. 2009.

[5] A. G. Yepes, F. D. Freijedo, and J. D. Gandoy, "Effects of discretization methods on the performance of resonant controllers,” IEEE Trans. Power Electron., Vol. 25, No. 7, pp. 1692-1712, Jan. 2010. 
[6] M. J. Newsman and D. G. Holmes, "Delta operator digital filters for high performance inverter applications," IEEE Trans. Power Electron., Vol. 18, No. 1, pp. 447-454, Jan. 2003.

[7] P. Roncero-Sanchez, E. Acha, J. E. Ortega-Calderon, and V. Feliu, "A versatile control scheme for a dynamic voltage restorer for power quality improvement," IEEE Trans. Power Del., Vol. 24, No. 1, pp. 227-248, Jan. 2009.

[8] V. F. Montagner and S. P. Ribas, "State feedback control for tracking sinusoidal references with rejection of disturbances applied to ups systems,” IECON '09. 35th, pp. 1764-1769, Nov. 2009.

[9] K. Zhang, Y. Kang, J. Xiong, and J. Chen, "Direct repetitive control of SPWM inverter for UPS purpose," IEEE Trans. Power Electron., Vol. 18, No. 3, pp. 784-792, May 2003.

[10] G. Escobar, A. A. Valdez, J. Leyva-Ramos and P. Mattavelli, "A repetitive-based controller for UPS using a combined capacitor/load current sensing," Power Electronics Specialists Conference, pp. 955-961, Jun. 2005.

[11] G. Escobar, A. A. Valdez, and R. E. Torres-Olguin, “A repetitive-based controller in stationary reference frame for D-Statcom in unbalanced operation," IEEE International Symposium on Ind. Electron., pp. 1388-1393, Jul. 2006.

[12] G. Escobar, P. R. Martinez, and J. Leyva-Ramos, “Analog Circuits to Implement Repetitive Controllers with Feedforward for Harmonic Compensation," IEEE Trans. Ind. Electron., Vol. 54, No. 1, pp. 567-573, Feb. 2007.

[13] P. C. Loh, Y. Tang, F. Blaabjerg, and P. Wang, "Mixed-frame and stationary-frame repetitive control schemes for compensating typical load and grid harmonics," IET. Power Electron., Vol. 4, No. 2, pp. 218-226, Feb. 2011.

[14] I. S. Gradshteyn and I. M. Ryzhik, "Table of integrals, series and products,” Academic Press, 6th Ed., 2000.

[15] D. N. Zmood, D. G. Holmes, and G. H. Bode, "Frequency-domain analysis of three-phase linear current regulators” IEEE Trans. Ind. Applicat., Vol. 37, No. 2, pp. 601-610, Mar./Apr. 2001.

[16] C. Cosner, G. Anwar, and M. Tomizuka, "Plug in repetitive control for industrial robotic manipulators," Proc. IEEE Int. Conf. Robot. Automat, pp. 1970 1975, May 1990.

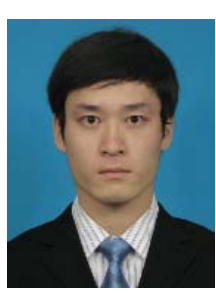

Guofei Teng was born in Shaanxi Province, China, in 1984. He received his B.S. and M.S. from the School of Electrical Engineering, Xi'an Jiaotong University, Xi'an, China, in 2007 and 2010, respectively. He is currently working toward his Ph.D. at the Power Electronics and Renewable Energy Research Center, Xi'an Jiaotong University. His current research interests include power quality control and the control of photovoltaic inverters.

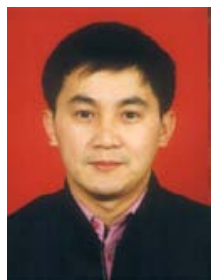

Guochun Xiao was born in Sichuan Province, China, in 1965. He received his B.S., M.S. and Ph.D. from the School of Electrical Engineering, Xi'an Jiaotong University, Xi'an, China, in 1987, 1990 and 2002, respectively. From 1990 to 1998, he was an Engineer at the Xi'an Electric Furnace Research Institute, Xi'an, China. Now he is an Associate Professor at Xi'an Jiaotong University. His current research interests include power conversion systems, harmonics suppression, reactive power compensation, and active power filters.

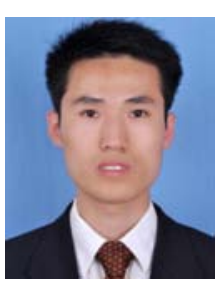

Leilei Hu was born in Jiangsu Province, China, in 1986. He received his B.S. in Electrical Engineering from the China University of Mining and Technology, Xuzhou, China, in 2009, and his M.S. from the School of Electrical Engineering, Xi'an Jiaotong University, Xi'an, China, in 2012. From 2009 to 2012, he was with the Power Electronics and Renewable Energy Research Center, Xi'an Jiaotong University, where he was a graduate student. Currently, he is working for the Sieyuan Electric Co., Ltd., as a Product Development Engineer. His current research interests include power electronics and power converters.

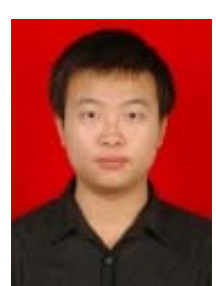

Yong Lu was born in Hunan Province, China, in 1989. He received his B.S. from the School of Electrical Engineering, Xi'an Jiaotong University, Xi'an, China, in 2010. He is currently a graduate student. His current research interests include power quality control and the control of inverters.

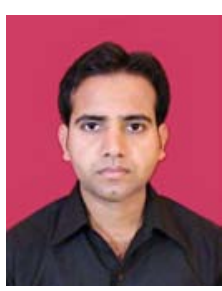

Yuba Raj Kafle was born in Chitwan District, Nepal in 1983. He received his B.E. from the Advanced College of Engineering and Management, Kathmandu, Nepal, in 2006. He is currently a graduate student in the School of Electrical Engineering, Xi'an Jiaotong University, Xi'an, China. His current research interests include power electronics, grid connected photovoltaic inverters, distributed power generation and power quality. 